\title{
Oscillations in the open solar magnetic flux with a period of 1.68 years: imprint on galactic cosmic rays and implications for heliospheric shielding
}

\author{
A. Rouillard ${ }^{1}$ and M. Lockwood ${ }^{1,2}$ \\ ${ }^{1}$ Department of Physics and Astronomy, University of Southampton, Southampton, SO17 1BJ, UK \\ ${ }^{2}$ Rutherford Appleton Laboratory, Chilton, OX11 0QX, UK
}

Received: 28 February 2003 - Revised: 1 September 2004 - Accepted: 5 October 2004 - Published: 22 December 2004

\begin{abstract}
An understanding of how the heliosphere modulates galactic cosmic ray (GCR) fluxes and spectra is important, not only for studies of their origin, acceleration and propagation in our galaxy, but also for predicting their effects (on technology and on the Earth's environment and organisms) and for interpreting abundances of cosmogenic isotopes in meteorites and terrestrial reservoirs. In contrast to the early interplanetary measurements, there is growing evidence for a dominant role in GCR shielding of the total open magnetic flux, which emerges from the solar atmosphere and enters the heliosphere. In this paper, we relate a strong 1.68year oscillation in GCR fluxes to a corresponding oscillation in the open solar magnetic flux and infer cosmic-ray propagation paths confirming the predictions of theories in which drift is important in modulating the cosmic ray flux.
\end{abstract}

Key words. Interplanetary physics (Cosmic rays, Interplanetary magnetic fields)

\section{Introduction}

The energy and composition spectra of Galactic Cosmic Rays (GCRs) provide unique information on astrophysical processes, but interpretation is complicated by the effects of magnetic fields which influence the particle's trajectory, particularly within the heliosphere (e.g. Ginzburg, 1996). At Earth, GCRs (and the secondary products generated when they hit the atmosphere) can deposit significant charge in small volumes of semiconductor to cause malfunctions in the avionics of spacecraft and aeroplanes (e.g. Dyer and Truscott, 1999). In addition, the implications for human health of prolonged exposure to cosmic rays in highaltitude aircraft has been the focus of recent study (Shea and Smart, 2000). GCRs also generate conductivity in the sub-

Correspondence to: A. Rouillard

(apr@ soton.ac.uk) ionospheric gap, allowing current to flow in the global electric thunderstorm circuit (e.g. Harrison, 2003) and it has been suggested in recent years that they influence the production of certain types of cloud with considerable implications for climate (Marsh and Svensmark, 2000). The spallation products of GCRs hitting atomic oxygen, nitrogen and argon in the Earth's atmosphere (cosmogenic isotopes which are subsequently stored in reservoirs, such as tree trunks, ocean sediments and ice sheets) are often used as indicators of solar variability in paleoclimate studies (e.g. Bond et al., 2001; Neff et al., 2001), although the implied links between solar irradiance variations and cosmic ray shielding by the heliosphere are not yet understood (Lockwood, 2002a, b). In all these studies, understanding how the heliosphere influences GCR fluxes and spectra is of key importance.

The modulation of GCRs is described by Parker's transport equation (Parker, 1965) which may be written for the phase space density, $f(r, p, t)$, as:

$$
\frac{\partial f}{\partial t}=\frac{\partial}{\partial x_{i}}\left[\kappa_{\mathbf{i j}} \frac{\partial}{\partial x_{j}}\right]-\boldsymbol{U} \cdot \nabla f-\boldsymbol{V}_{\boldsymbol{d}} \cdot \nabla f+\frac{1}{3} \nabla \cdot \boldsymbol{U}\left[\frac{\partial f}{\partial \ln p}\right]+Q,
$$

where the terms on the right-hand side correspond to diffusion, convection, particle drift, adiabatic cooling or heating and any local source $Q . \kappa_{i j}^{S}$ is the symmetric diffusion coefficient, $U$ the outward solar wind velocity.

It has been argued (Fisk, 1999; Moraal, 1999) that the theory of cosmic ray transport in the heliosphere is now probably complete and the greatest challenge in recent years has been mainly to evaluate the magnitude, spatial and energy dependence of the different terms and the parameters they depend on. Our present understanding of the contributions of these various terms in Eq. (1) to the overall modulation, has been obtained through theoretical estimations of the different modelled parameters and comparison to the limited data available, in particular, deductions made from observed particle energy spectra. In this way, the modulation effects of outward convection and adiabatic energy losses in the solar 
wind speed have become well understood and therefore pose no major problems or uncertainties in modelling, especially since the Ulysses mission has provided us with information from outside the ecliptic plane (Goldstein, 1994). Similarly drift effects in the smooth background field are also well understood, even in discontinuous media, such as the heliospheric current sheet near the ecliptic plane or near the heliosphere's boundary. The problems arise mainly from the great uncertainties remaining about the effect of irregularities in the magnetic field on drifts and our great lack of understanding of the scattering of charged particles parallel and perpendicular to the interplanetary field (IMF) due to magnetic field irregularities (Moraal, 1999). These limitations imply major inherent assumptions for any GCR modulation model. In an attempt to gain a better understanding of the diffusion mechanisms, an "ab-initio" theory has been developed (Parhi, 2001), in which the diffusion coefficient $\kappa_{i j}^{S}$ is derived from first principles. This "ab-initio" approach relates the diffusion term to charged particle scattering in complex space-time dependent magnetoplasma turbulences. This description predicts a good correlation between the charged GCRs propagation and heliospheric magnetic field variations, which was recently observed with solar particles (Dröge, 2003).

The motivation behind this work was raised by the recent discovery of simple and significant anti-correlations between the flux of GCR particles measured by terrestrial neutron monitors and the magnitude of the heliospheric field at Earth (Cane et al., 1999; Belov, 2000) and its radial component which is proportional to the open solar flux (Lockwood, 2001, 2003). As a consequence of this, recent works in the literature have used these anti-correlations to investigate the effect of solar modulation of GCRs using simpler concepts than the full Parker equation. For example, Wibberenz et al. (2002) assumed that the radial diffusion coefficient scales as some power of the magnitude of the IMF and invoked continuous recovery processes (related to particle entry into depleted regions of the heliosphere by drift and diffusion), to develop a simple model which seems to map cosmic ray intensity variations very well over the last four solar cycles (Wibberenz and Cane, 2000; Wibberenz et al., 2002). In their model, the initial cosmic ray intensity, assumed to be a steady-state solution of a spherically symmetric approximation, is perturbed by increases in the IMF that propagate away from the Sun and cause a reduction in the GCR radial diffusion coefficient. The assumed inverse coupling of the IMF with cosmic ray spatial diffusion coefficients is consistent with the concept of propagating diffusive barriers first introduced theoretically by Perko and Fisk (1983). The flux decrease associated with these barriers is followed by a recovery caused by both diffusion mechanisms and the large-scale influence of drifts. Longer recovery times are therefore expected for periods of $A<0$ when particle inflows are along the heliospheric current sheet than for $A>0$, where inflows are expected from over the poles. The recent work by Ferreira et al. (2003) to include the interplay between these diffusive barriers and large-scale drifts in a full time-dependent model has shown very promising results concerning the charge-sign dependent modulation effects predicted by drift theory. The model used is based on a numerical solution of Parker's time-dependent transport equation Eq. (1), and the diffusion coefficient is assumed to be proportional to the solar magnetic flux $B^{-n}$. It should be noted that while at neutron monitor rigidities the diffusion coefficient works best with a direct inverse relationship, $n=1$, for the lower rigidities ( $<5 \mathrm{GV}$ ) even values of $n=3$ do not reproduce the required solar cycle amplitude change, as demonstrated by Potgieter and Ferreira (2001). These authors showed that a time-varying $n$ (over the solar cycle) should be used and the tilt, $T$, of the Heliospheric Current Sheet (HCS) was equated to $n$ in the simple form $n=T / T o$, where $T o=11$.

Recent work has shown that a better understanding of the relationship between the evolving open solar magnetic field and the variation of the cosmic ray intensity as measured by neutron monitors at Earth is fundamental to cosmic ray modulation theory. In this paper we present further common features of the total open solar flux and GCR variations in both the time and frequency domains and look at the implications for where and how GCRs are shielded away from the Earth.

\section{The open solar magnetic flux estimates}

Three methods have been devised, to date, to estimate the open solar flux. The most direct method for computing open solar flux has been made possible by the discovery by the Ulysses spacecraft that the radial component of the heliospheric field is independent of heliographic latitude (Smith and Balogh, 1995; Balogh, 1995; Lockwood et al., 1999b). This discovery has been explained in terms of the low plasma $\beta$ of the expanding solar wind at around $1.5 R_{S}<r<10 R_{S}$, where slightly non-radial flow allows the magnetic flux to redistribute itself, to give latitude-independent tangential magnetic pressure and thus a uniform radial field component (Suess and Smith, 1996). Because of this result, the radial field seen near Earth $B_{r 1}$ can be used to compute the total flux threading a heliospheric sphere of radius $R_{1}=1 \mathrm{AU}$ :

$[F s]_{\mathrm{IMF}}=4 \pi R_{1}\left|B_{r 1}\right| / 2$.

The factor 2 arises because half the flux through this surface is outward and half is inward. Lockwood et al. (2004) have shown that errors in using (2) are less than 5\% for averages on time scales greater than a 27-day solar rotation period. Observations of the IMF magnitude have been made since 1963. The earlier data were intercalibrated into the homogeneous "Omnitape" data set of hourly data by Couzens and King (1986), and this has been extended with data for up to the present day. This data set gives the near-Earth heliospheric radial field component $B_{r 1}$ which can be used with Eq. (2) to give the total open solar flux.

A second method was developed by Lockwood et al. (1999a, b) and Lockwood and Stamper (1999) and uses the $a a$ index, devised by Mayaud (1972), to quantify geomagnetic activity from a data series that extends 
homogeneously and continuously back to 1868. A description of the procedure has been given previously (Lockwood et al., 1999a, b; Lockwood and Stamper, 1999) and will not be repeated here. However, we would like to stress the physical assumptions used. The method is based on the theory of solar-wind magnetosphere energy coupling by Vasyluinas et al. (1982) which has shown to be the most successful by Finch et al. (submitted, 2002) ${ }^{1}$, giving a correlation of 0.97 between interplanetary parameters and the $a a$ index on annual time scales. Secondly, it applies the Parker spiral theory to the ecliptic heliospheric field which, on the annual time scales used, matches the data exceptionally well (Gazis, 1996; Stamper et al., 1999). Thirdly, the method exploits our understanding of the role of fast solar wind streams in generating recurrent geomagnetic activity (Cliver et al., 1996; Hapgood, 1991). The three correlations used to generate the required coefficients are all over 0.95 and are all more than 99.99\% significant, allowing for the persistence in the various data series. The open solar flux derived by this method is here termed $[F s] a a$ (and is compared to $[F s]_{\mathrm{IMF}}$ in Fig. 1).

The third method uses solar surface magnetograms, which give the line-of-sight component of the photospheric magnetic field. This is mapped up to a hypothetical surface called the "coronal source surface" where the field is purely radial. In order to do this, it is assumed that there are no currents in the corona between the photosphere and the source surface where the field is corrected to be radial. The source surface is also assumed to be spherical at a heliospheric distance of $r=2.5 \mathrm{Rs}$. Although a very useful, idealised concept, there is no a priori reason why this surface should be spherical, indeed, it may not exist at all. Thus, although this "potential field source surface" (PFSS) gives a useful indication of the distribution of the photospheric footprints of the open flux, there are still uncertainties in the estimated total open solar flux, $[F s]_{\text {PFSS }}$, because of the assumptions required.

Figure 1 shows a comparison of $[F s] a a$ and $[F s]_{\mathrm{IMF}}$ at Earth. The correlation between both is 0.74 which is significant at the $90.5 \%$ level. The best correlation is forced with the $[F s] a a$ advanced by a lag of $0.74 \mathrm{yr}$. Note that although $[F s] a a$ estimates have been made on a monthly basis, they are based on a full year of data, centred on the month in question. Thus, the $[F s] a a$ data have inherent smoothing which may account for much of this best-fit lag (which has an uncertainty of $0.33-1.03$ yrs, computed using Fisher-Z test as implemented by Lockwood, 2002a). The longest period of disagreement between both time series occurs between 1967.5 and 1970.

\subsection{Four solar cycles of anti-correlation between $[F s] a a$ and high energy GCR counts}

The anti-correlation of the open solar flux, as estimated from the radial component of IMF, $[F s]_{\mathrm{IMF}}$ with the count rate

\footnotetext{
${ }^{1}$ Finch, I., Lockwood, M., and Stamper, R.: Solar windmagnetosphere coupling functions on timescales of 1 day to 1 year, Ann. Geophys., submitted, 2002.
}

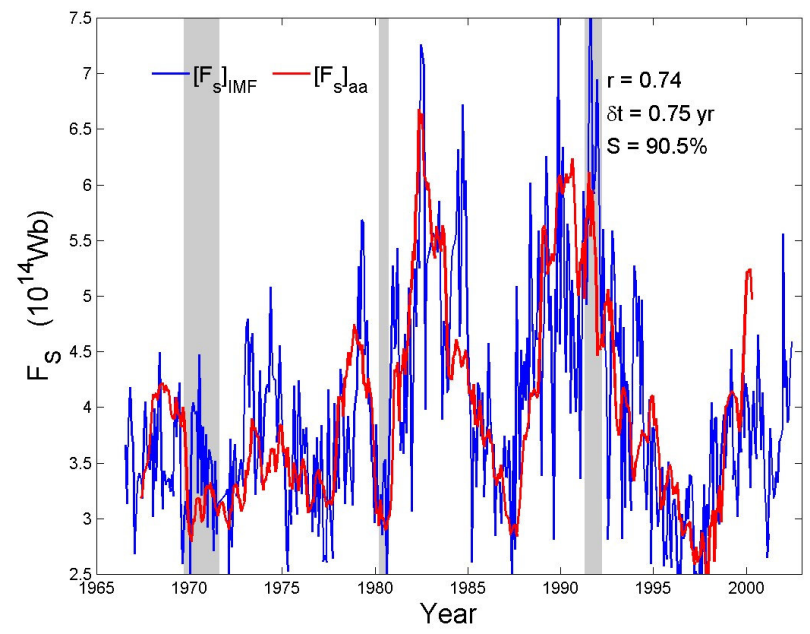

Fig. 1. Comparisons of the monthly open solar flux estimates derived from the radial component of the IMF and from the $a a$ geomagnetic index ([Fs] $\mathrm{IMF}$ and $[F s] a a$, respectively, in blue and red). The grey bands mark the times of the reversal in the solar polar field by showing the times between the polar field reversals in the two solar hemispheres. The correlation coefficient is $r=0.74$, which is significant at the $S=90.5 \%$ level. The peak correlation is obtained at a lag $\delta t=0.75 \mathrm{yr}$ (defined as positive with $[F s]_{\mathrm{IMF}}$ leading $[F S] a a)$ with an uncertainty range $0.33-1.08$ yr. The $[F s] a a$ data sequence is plotted using the best-fit lag $\delta t$.

of GCR particles of rigidity exceeding $13 \mathrm{GV}$, was evaluated by Lockwood (2003). The correlation coefficient for the full interval of coincident data (1968-2001) was $c=-0.61$. Allowing for the persistence in both the $H$ and the $[F s]_{\mathrm{IMF}}$ data series, the significance of this correlation, $S$ exceeded $99.999 \%$. The anti-correlation was similar to that reported by Cane et al. (1999) and Belov (2000), who used the strength of the near-Earth $B$ rather than its radial component $B_{r}$ : this was to be expected because the Parker spiral angle (averaged over these monthly intervals) remains approximately constant, such that $B_{r}$ is highly correlated with $B$ (Lockwood et al., 1999b). In fact, the anticorrelation with $B_{r}$ was slightly the strongest of the two, but using a Fischer- $Z$ test this difference was not found to be statistically significant. Because it has a longer data series and because there are uncertainties in the early $[F s]_{\mathrm{IMF}}$ data, the $[F s]_{a a}$ data are very valuable for GCR shielding studies. The grey histogram in Fig. 2 shows the time-variation of the total open solar magnetic flux estimate, $[F s] a a$, derived from the aa geomagnetic index using the procedure of Lockwood et al. (1999a, b). The black line shows $H_{\text {FIT }}$ the best linear regression fit to [Fs]aa of GCR count rate $\mathrm{H}$ observed by Hawai/Huncayo neutron monitors which together give a continuous and homogeneous data series of GCR count rates of rigidity exceeding $13 \mathrm{GV}$. The inverse of the GCR counts map the open solar flux very closely. We notice, for example, that $[F s] a a$ peaks shortly after each sunspot maximum when $H$ is a minima. Furthermore, the rounded and the peak-shaped minima in $[F s] a a$ match well the shapes of the peaks in $H$ and various well-correlated and 


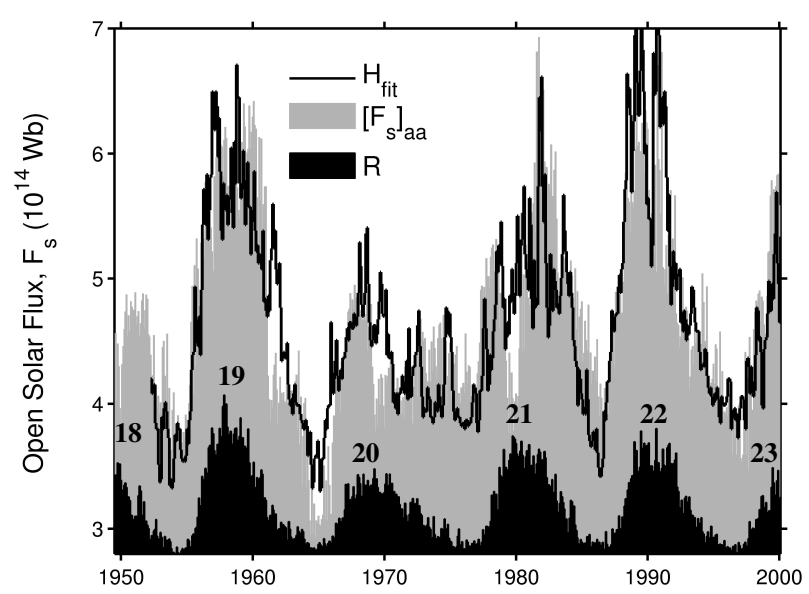

Fig. 2. Variations of monthly means of solar, heliospheric and cosmic ray data since 1950 . The grey histogram gives the open solar magnetic flux $[F s] a a$, deduced from the $a a$ geomagnetic index using the method of Lockwood et al. (1999a, b). The black line is $H_{\text {FIT }}$, the best fit of the anti-correlated cosmic ray counts $H$, observed by the equatorial Huancayo and Hawaii neutron monitors (which form a homogeneous data sequence on positively-charged GCRs with rigidities exceeding $13 \mathrm{GV}$ ). The black histogram gives the sunspot number $R$ for comparison.

short-lived peaks, and minima appear in both $[F s] a a$ and $H_{\text {FIT }}$. Monthly averages of $[F s] a a$ and $H$ give a correlation coefficient $r$ of $=-0.777$ which means that $60 \%$ of the variation in the cosmic ray variation is explained (in a statistical sense) by the open solar flux. Allowing for the persistence in both the $H$ and the $[F s] a a$ data series, the significance of this correlation, $S$ exceeds $99.999 \%$ (i.e. there is less than a $0.001 \%$ probability that this result was obtained by chance).

2.2 Disagreements between the estimates of the open flux and cosmic ray counts at Earth in solar cycle 20

As we can see in Fig. 2 the main differences between the variations of $[F s] a a$ and the best fit cosmic ray fluxes, $H_{\mathrm{FIT}}$ occur on the descending phase of solar cycle 19 and throughout the anomalous solar cycle 20. It is interesting to note that initial studies did not find a good correlation between GCRs and the IMF (Hedgecock, 1975) but more recent data show the IMF to be a more dominant factor than, for example, the tilt of the heliospheric current sheet (which is itself well correlated with Fs) (Cane et al., 1999; Belov, 2000). Here we report similar but less significant disagreements between $H$ and $[F s] a a$ in the same period of time. Figure 1 shows that $[F s] a a$ goes though a maximum in the ascending phase of solar cycle 20 that is not reflected in $[F s]_{\mathrm{IMF}}$ (cf. Fig. 1) but is seen in a corresponding minimum in the cosmic ray counts (cf. Fig. 2). Additionally, in the descending phase of cycle 20 (1971-1975), $[F s]_{\mathrm{IMF}}$ goes through a maximum neither reflected with the same proportion in [Fs]aa nor $H$.

\subsection{The 22-year periodicity}

The observed GCR fluxes show a marked 22-year cycle, with alternate peaked and rounded maxima (which, because $H$ is anti-correlated with $[F s] a a$ appear as $\mathrm{V}$ - and U-shaped minima of $H_{\text {FIT }}$ in Fig. 2). This has been attributed to the effect of the drift term in the transport equation which has been postulated to dominate the GCR shielding for 6-7 years around solar mininum (the quiet heliosphere), the differences between odd and even numbered cycles arising from the fact that the heliospheric field polarity A reverses shortly after each sunspot maximum: U- and V-shaped GCR maxima are expected for $q A>0$ and $q A<0$, respectively (Jokipii et al., 1977; McDonald et al., 1993). At solar maximum the paradigm of the quiet heliosphere (dominated by the effects of the guiding centre, gradient and curvature drift motions and quasi-steady corotating interaction regions CIRs) does not apply. In this diffusive mode of sunspot maximum, transient events are thought to dominate and while drifts are thought to have still high magnitudes they are less globally coherent.

Figures 1 and 2 show that alternate $\mathrm{U}$ - and $\mathrm{V}$-shaped minima are also seen in both $[F s] a a$ and $[F s]_{\mathrm{IMF}}$, implying that a 22-yr cycle was also a feature of the open solar magnetic flux emergence. It appears therefore that on top of a twentytwo-year pattern in the polarity of the Sun's magnetic field, a twenty-two-year pattern is also seen in the time varying quantity of open field lines threading a sphere centred on the Sun and passing at $1 \mathrm{AU}$. This correspondence could follow naturally from an eleven-year variation in the cosmic ray flux resulting from a series of outward propagating transients, or even merged transients as first identified by McDonald et al. (1981) but not from a drift-induced 11-year modulation as proposed by Jokipii et al. (1977). The presence of charge sign dependent effects around the years of solar minimum, 1995.5-1998, has been recently demonstrated convincingly by Heber et al. (1999), using Ulysses Kiel Electron Telescope data. Galactic comic electrons also show this $22-\mathrm{yr}$ cycle and, having the opposite charge $q$, the drift theory predicts that this should be in antiphase with the nucleon GCR variation (Evenson, 1998). Measurements of $2.5 \mathrm{GV}$ protons and electrons during the first fast latitudinal scan (1994.71995.6) revealed a small but clear latitudinal gradient in the time series not observed in the electron data (Heber et al., 1999) and in agreement with a drift-mode according to Ferreira et al. (2003) (where protons are expected to drift in from the poles during these years of $A>0$ ). In the same paper Heber et al. showed that between 1995.5 and 1998 the electron time series presented greater sensitivity to variations in the tilt of the HCS than the proton time series in agreement with negative particles propagating inward along the current sheet (Heber et al., 1999). These results have revealed charge-sign dependent effects in the years with an extrapolated HCS tilt smaller than 20 degrees and do not allow any conclusions to be drawn on their direct relevance to the 11year modulation effect. The more recent Ulysses KET data recorded during the global solar polarity change was also 
analysed and shows a charge-sign dependence before and after the years of solar maximum (Heber et al., 2003). Heber et al. (2003) noticed that the relative variation of the electron and proton counts along the Ulysses orbit phased in for two and a half years at solar maximum but phased out outside this period in the transition years from solar maximum to solar minimum mode. The most promising direct detection of a charge-sign dependent effect at solar maximum was obtained by Clem and Evenson (2002), using the positron abundance recently measured by the LEE/AESOP payload (Low Energy Electrons/Anti-Electron Sub Orbital Payload) aboard a balloon flight. As seen in Fig. 5 of their paper a clear transition in the abundance of positron at Earth is seen during polarity reversal. The result is based, however, on one set of measurements in the new $A<0$ polarity state, and new measurements are needed to obtain the average abundance of the current polarity state. These new results obtained in the years before, during and just after solar maximum suggest a possible important role of charge-sign dependent effects in the years of transition from GCR minimal to maximal values. As mentioned earlier Wibberenz et al. (2002) have combined these drift-associated effects and the time-dependent transient modulators by postulating that the background drifts set the global distribution of cosmic rays in the heliosphere which is then modulated by the outwardly propagating transients. We are lacking, however, observations that would allow for a proper determination of the relative role of drifts and propagating diffusive barriers in generating the 11-year cosmic ray cycle. The close correspondence between the various open flux estimates and the cosmic ray flux at Earth in the last two solar cycles is striking and could reveal fundamental properties of the photospheric origin and low corona generating/redistribution mechanisms of both the solar open magnetic field lines and their associated GCR modulators. We also think that a comparison of the anomalous cosmic ray cycle, a period when the open flux does not follow the GCR flux as well (solar cycle 20), with the two recent solar cycles, could reveal interesting features on the origin of the 11-year cosmic ray cycle and perhaps put this 11-year open flux-GCR correspondence in context with recent results on charge-sign dependence. These claims will be addressed thoroughly in a forthcoming article.

Here we investigate the two possibilities that the 22-yr cycle in GCRs may result from the polarity dependent drifts or that it is related to open flux emergence from the Sun.

Section 3 discusses corresponding periodicities detected in the large-scale magnetic field and their relation to the solar surface dynamics with periodicities observed in cosmic rays counts. Section 4 presents more correlative work between the finer time series structure of the open flux and cosmic ray counts measured at Earth. Section 5 presents quantitative work on the lags observed between the fluctuations of the solar magnetic field and cosmic ray measurements at $1 \mathrm{AU}$.

\section{Periodicities in the large-scale magnetic field and in cosmic ray counts at Earth}

\section{$3.1 \quad 1-2$-year quasi-periodicities}

The Sun's large-scale magnetic field and its proxies are known to undergo substantial variations on time scales much less than a solar cycle but longer than the 27-day rotation period. For instance, Gnevyshev argues that each solar cycle exhibits two peaks of maximum activity separated by $2-3 \mathrm{yr}$, a feature present in large sunspots, major flares, coronal green-line emission and geomagnetic activity (Gnevyshev, 1967, 1977). Storini et al. (1997) detected double-peaked structures in the cosmic ray flux during solar cycles 19-22, with intervening "gaps" coinciding with the time of heliomagnetic polarity reversal. Although the effects described by Gnevyshev and subsequent authors are related to variations in the Sun's large-scale magnetic field occuring at and just after sunspot maximum, flucutations on time-scales of 1-3 yr are also present at other phases of the solar cycle appearing from time to time and have been detected in various solar wind parameters (Richardson, 1994), as well as auroral records (Silvermann and Shapiro, 1983). Recently, Lockwood (2001) has suggested that the field variations of $\sim 1$ year in duration associated with medium-term events may be related to the oscillations close to the base of the solar convection zone observed by the SOHO spacecraft (Howe et al., 2000). In fact, a recent study suggests that periodicities of 1.3-1.4 yr may occasionally appear from 1-yr decaying stochastic processes associated with the emergence of active regions on the solar surface (Wang and Sheeley, 2003).

\subsection{The imprint of the open solar flux in GCR fluxes}

A strong oscillation in GCR fluxes of period $T=1.68 \mathrm{yr}$ (frequency, $f=0.595 \mathrm{yr}^{-1}=9 \mathrm{nHz}$ ) has been reported recently and related to similar oscillations in solar surface features (Valdés-Galicia et al., 1996; Valdés-Galicia and Mendoza, 1998). This periodicity has also been found in the coronal hole area (McIntosh et al., 1992; Maravilla et al., 2001). Here we remove the dominant solar cycle periodicity using a highpass filter $\left(f>0.2 \mathrm{yr}^{-1}, T<5 \mathrm{yr}\right)$ and use non-parametric and parametric spectral estimations to investigate this oscillation. In addition to application to the GCR data series, these methods were applied to the open solar flux derived from both solar surface magnetograms by the PFSS (Potential Field Source Surface) method (Wang and Sheeley, 1995) and the near-Earth IMF observations (Lockwood, 2003).

Results of a non-parametric spectral estimation of the filtered time series, made using Fourier analysis, are shown in Fig. 3. For this 22 -year period (1972-1994) the 1.68-year oscillation is the strongest feature in the power spectra of both GCR fluxes and of the open flux, once the dominant 11-year solar cycle is suppressed by the filter. For this 22-yr interval, power spectra have a frequency resolution of $0.048 \mathrm{yr}^{-1}$. The spectra are offset vertically for clarity. From bottom to top they are for: the Climax neutron monitor ( $>3 \mathrm{GV}$ ) 


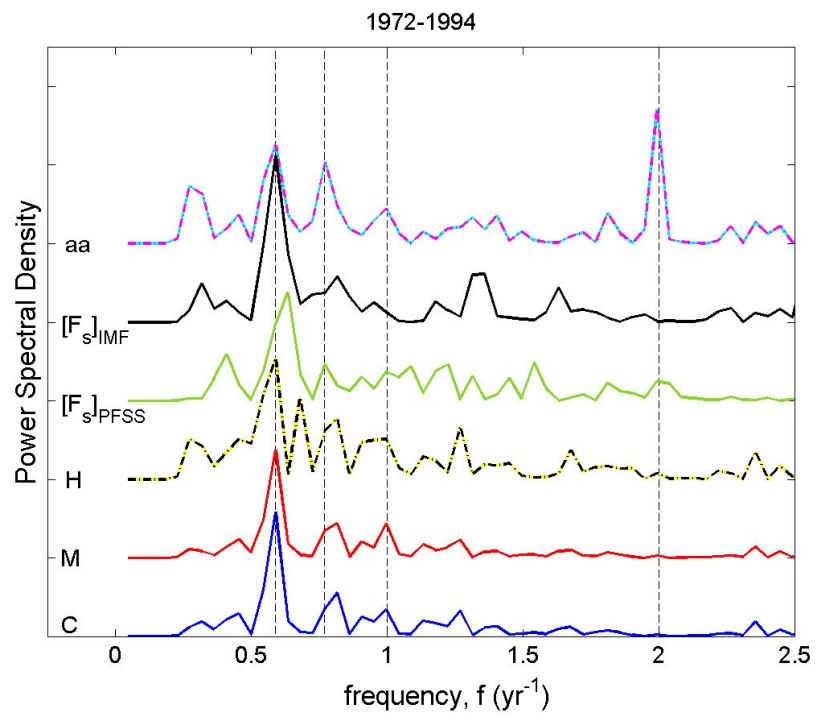

Fig. 3. Stacked power spectra for 1972-1994. All data have been Fourier analysed after being passed through a high pass filter $\left(f>0.2 \mathrm{yr}^{-1}\right)$ to suppress the solar cycle variation. From bottom to top: Climax neutron monitor ( $>3 \mathrm{GV}$ ) counts, $C$ (blue); Moscow neutron monitor ( $>4 \mathrm{GV}$ ) counts, $M$ (red); Huancayo/Hawaii neutron monitor ( $>13 \mathrm{GV}$ ) counts, $H$ (black and yellow dashed); open solar flux deduced from photospheric magnetograms by the PFSS method, $[F S]_{\text {chem PFSS }}$ (green); open solar flux deduced from the radial interplanetary magnetic field measured near Earth, $[F s]_{\mathrm{IMF}}$ (black); the $a a$ geomagnetic index (light blue and mauve). Vertical dashed lines mark periods of $1.68 \mathrm{yr}, 1.30 \mathrm{yr}, 1.00 \mathrm{yr}$ and $0.50 \mathrm{yr}$.

counts, $C$ (blue); the Moscow neutron monitor ( $>4 \mathrm{GV}$ ) counts, $M$ (red); the Huancayo/Hawaii neutron monitors (>13 GV) counts, $H$ (black and yellow dashed); the open solar flux deduced from photospheric magnetograms by the PFSS method, $[F s]_{\text {PFSS }}$ (green); the open solar flux deduced from the radial interplanetary magnetic field measured near Earth, $[F s]_{\text {IMF }}$ (black); and the aa geomagnetic index (light blue and mauve). The vertical dashed lines mark the frequencies corresponding to period $T$ of $1.68,1.3,1.0$ and 0.5 years. All spectra show a peak at $T=1.68 \mathrm{yr}$, except for $[F s]_{\text {PFSS }}$ which shows enhanced power at this period but peaks for the adjacent quantised period of $1.54 \mathrm{yr}$. Some spectra also show a peak at $T=1.3 \mathrm{yr}$ which is seen in solar wind speed and geomagnetic activity (Richardson et al., 1994; Mursula and Zieger, 2000; Lockwood, 2001). The $a a$ geomagnetic activity index also shows a strong peak at $0.5 \mathrm{yr}$, arising from the effect of the Earth's axial tilt on coupling between the solar wind and the Earth's magnetosphere (Russell and McPherron, 1973). Given the strong equinoctial effect that this mechanism causes in geomagnetic activity, it is interesting to note that the power in the variation at $T=0.5 \mathrm{yr}$ is only $50 \%$ bigger than that in $a a$ at $T=1.68 \mathrm{yr}$. The $a a$ index also shows a weak peak at $1.0 \mathrm{yr}$ which is most likely a result from ionospheric conductivity variations at the two magnetometer sites used to compile the index. Weak annual variations are also seen in the cosmic ray data from all the sites employed: these indicate that either the corrections to allow for the effect of atmospheric pressure on raw neutron counts have not completely suppressed the seasonal variation introduced by this effect, or there is a strong yearly effect of the inclination of the Earth (Nagashima et al., 1985).

The parametric spectral estimation was also made using the maximum entropy method (MEM). The time series is modelled parametrically and then processed with the condition that the entropy of the original random time series is maximised (Kay and Marple, 1981). This has the effect of "flattening" the power spectrum and can provide a frequency spectrum with sharp spectral features. MEM can be unreliable if the order of the parametric model used is too small or too big: too small a model order will give a spectrum with bad resolution, too big a model order can lead to a spectrum with "artificial peaks" (with some spectral line splitting occurring). Some criteria of model order selection were used to give an idea as to what model order should be selected for a particular time series. The MEM procedure was applied to the filtered Climax neutron monitor counts, $C$, the open solar flux data deduced from surface magnetograms by the PFSS method, $[F s]_{\text {PFSS }}$, and to the open solar flux deduced from the radial interplanetary field measured at Earth, $[F s]_{\mathrm{IMF}}$. As for the Fourier analysis, in all MEM spectra the variation at $T=1.68 \mathrm{yr}$ in the corresponding time series is the most important peak once the 11-year solar cycle variation is filtered out. This oscillation therefore appears to be a characteristic imprint of the open solar flux on the GCRs. Application of a band-pass filter $(5<T<1 \mathrm{yr})$ on $H$ and $C$, which would effectively remove the overall 11-yr solar cycle variation, would leave a time series $\Delta H$ and $\Delta C$ dominated by the $1.68-\mathrm{yr}$ oscillation in an almost synchronised way to $\Delta[F s]_{\mathrm{IMF}}$ with probable exact phase variations. We investigate this question in Sect. 4 by means of correlation analysis.

Figure 4 shows the variation of the power spectral densities of the cosmic ray count rates, two open solar flux estimates $(\Delta[F s] a a$ is not used because of the smoothing inherent in its derivation - see Lockwood et al., 1999a, b) and the tilt of the heliospheric current sheet. These are obtained from the Fourier analysis (using 11-year sliding data intervals) and plotted here in spectrogram format. The time of a particular power density is taken as the middle date of each 11-year window. An 11-year window was taken as a compromise between time and frequency resolution (windowing effects are discussed below), where the length of the window taken of course determines the starting and ending date of the various spectrograms. The peak around frequency $f=1 / 1.68 \mathrm{yr}^{-1}$ is a persistent and strong feature $\left(>10 \% 2 \mathrm{~Hz}^{-1}\right)$ in all spectrograms except for the tilt of the heliospheric current sheet which has for this frequency a power spectral density less than $6 \% \mathrm{~Hz}^{-1}$ and appearing much later in the solar cycle. We also notice that during the period where $f=1 / 1.68 \mathrm{yr}^{-1}$ appears strongest in all data sequences, the heliospheric current sheet reveals a frequency of $\sim 1 . / 0.72$ yrs not seen in the cosmic ray spectrograms corroborating the results of Cane et al. (1999), who found that the overall correlation between HCS tilt and cosmic ray count rates was low. The 

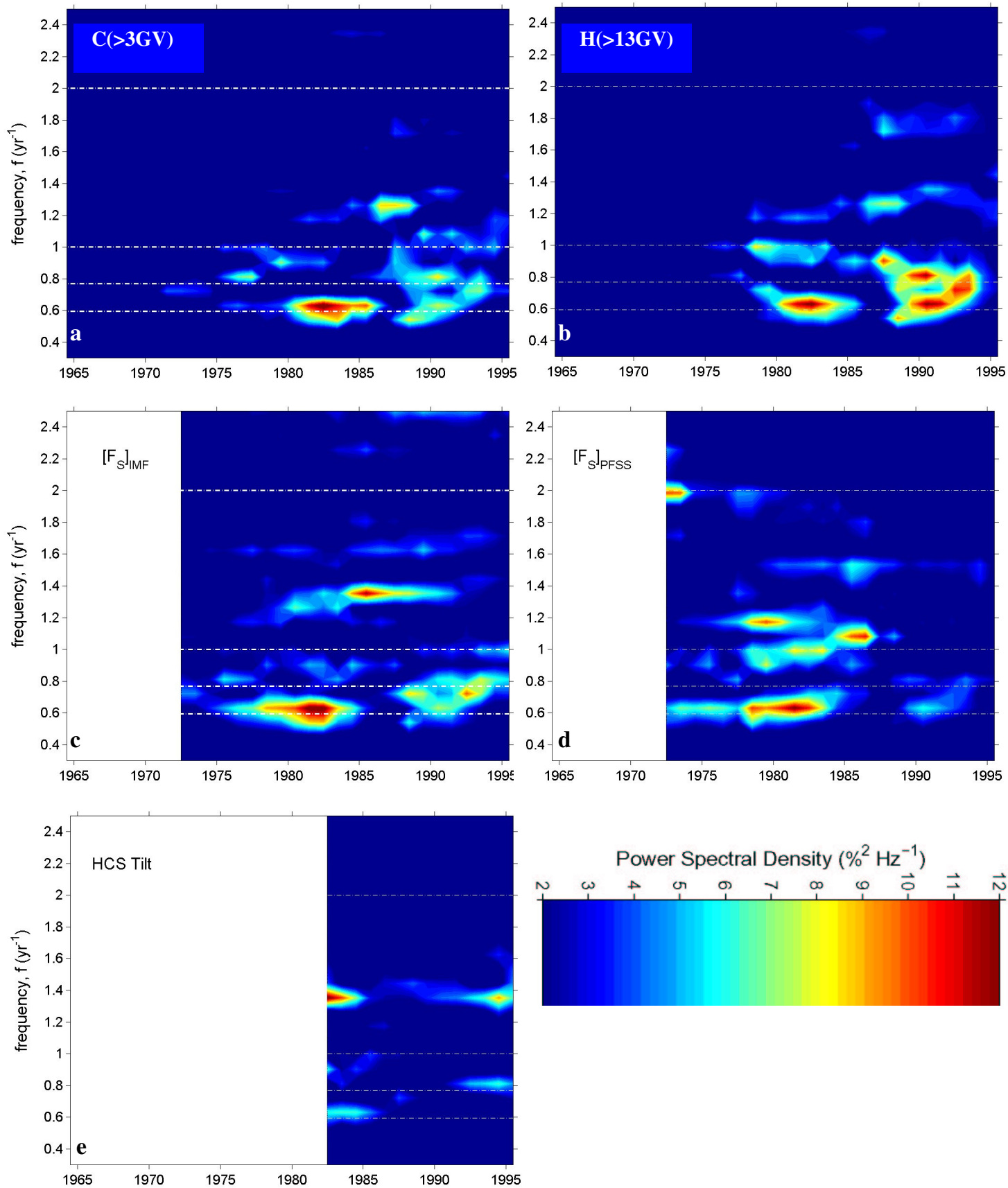

Fig. 4. Spectrograms showing contours of power spectral density as a function of date and frequency, $f$. The dashed white lines in each panel show periods of $0.5,1.0,1.33$ and 1.68 years. Power spectra are computed for 11-yr periods, centred on the dates shown on the horizontal axes. Plots are for: (a) Climax neutron monitor ( $>3 \mathrm{GV}$ ) counts, $C$; (b) Huancayo/Hawaii neutron monitor (>13 GV) counts, $H$; (c) open solar flux deduced from the radial interplanetary magnetic field measured near Earth, $[F s]_{\mathrm{IMF}}$; (d) open solar flux deduced from photospheric magnetograms by the PFSS method, $[F s]_{\mathrm{PFSS}}$; and (e) the heliospheric current sheet tilt angle.

correspondence between the spectrograms for the cosmic ray counts and the $\Delta[F s]_{\mathrm{IMF}}$ spectrogram is remarkable: most frequencies seen in Fig. 4c also appear in Figs. $4 a$ and $b$. The spectrograms are also quite similar to that obtained by Kudela et al. (2002) using wavelet transforms as their spectral method. However, the periodicity pattern highlighted by Mursula and Zieger (1999) is not reproduced clearly here. Figure 5 shows the time variations of spectral power at period
1.68 yr. Results are shown for GCR counts $C, M$ and $H$ (respectively, $>3 \mathrm{GV},>4 \mathrm{GV},>13 \mathrm{GV}$ ), the open solar flux estimates $[F s]_{\mathrm{IMF}}$ and $[F s]_{\mathrm{PFSS}}$ and the HCS tilt. Similar variations are seen in all parameters with peaks at the time of peak sunspot number (shown by the coloured histogram at the bottom of the plot); however, the peak in 1968 is proportionally weaker. The top panel uses 11-year sliding windows (the same as the spectrograms shown in Fig. 4), which 

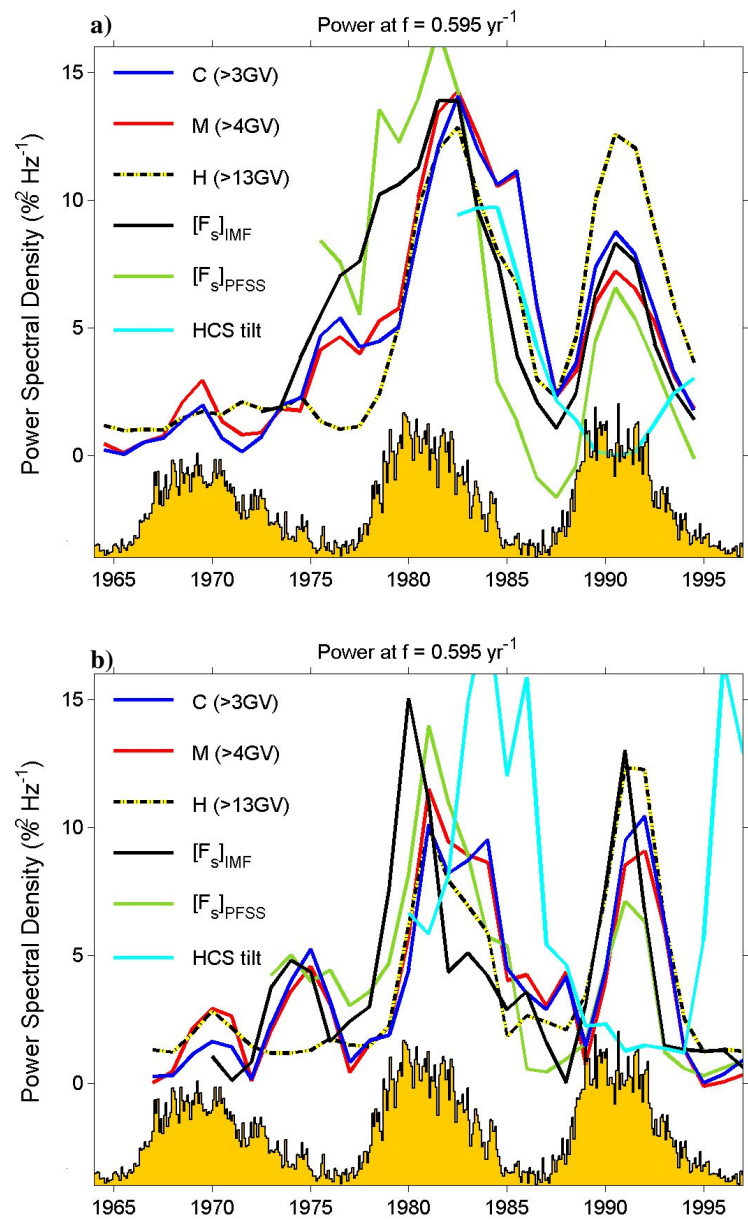

Fig. 5. (a) Time variations of the power spectral density around the period of 1.68 years $(1.55 \mathrm{yr} \leq T \leq 1.81 \mathrm{yr}$ ) from analysis of (top) 11-year segments of data and (b) 6-year segments of data. The variations are shown using the same line colour-coding scheme as Fig. 3. The sunspot number variation is shown for comparison. Power spectral densities have been scaled to that for the Climax counts, $C$, using the best least-squares linear regression fits of the time variations.

maintains high frequency resolution but is comparable to the solar cycle. In the lower panel we repeat using 6-year windows (the same as used in Figs. 7, 8, 9 and 10 described later) with lower frequency but higher time resolution. The time variations are, generally speaking, similar at sunspot maximum; although for the first cycle the smaller peak is in the declining phase.

\section{Procedure of the correlation analysis}

The presence of corresponding quasi-annual periodicities in both cosmic ray and open solar flux data motivated a more detailed correlative study. We present in this section the results of a window-by-window correlation analysis between the open solar flux estimates and the cosmic ray counts measured by the Climax and Huancayo/Hawaii neutron monitors
$C$ and $H$, respectively. Open flux estimates derived from geomagnetic activity and radial IMF observations, $[F s] a a$ and $[F s]_{\mathrm{IMF}}$ are both used. The correlation analysis of $[F s] a a$ with $H$ and $C$ is limited to their unfiltered data due to the time resolution of $[F s] a a>1 \mathrm{yr}$ but these data provide a longer overall interval. However, the correlation analysis of $[F s]_{\mathrm{IMF}}$ with $H$ and $C$ can be carried out on both the detrended data (after application of a band pass filter $5<T<1 \mathrm{yr}$ ) and on the raw data.

\subsection{Description of the window by window cross- correlation analysis}

To evaluate the time-variation of the cross-correlation coefficient between open solar flux estimates and the neutron monitor count rates at Earth over the last four completed solar cycles, we employ the cross-correlation analysis illustrated by Fig. 6 . This example shows a cross-correlation between $\Delta[F s]_{\mathrm{IMF}}$ and $\Delta C$, where the notation $\Delta X$ refers to parameter $X$ after passing through a high band-pass filter. The filter used has a pass-band of 4 yrs. FHWM is centred on 3 yrs. Here we filter 11-year segments of the data of which we then employ the middle 6 years, thereby avoiding end effects of the sliding window. Figure 6a shows an example of a temporal variation of $\Delta[F s]_{\mathrm{IMF}}$ (dashed line) and the best linear regression fit $\Delta C_{f i t}$ (solid line) and Fig. $6 \mathrm{~b}$ shows the same data in scatter plot format for the best-fit lag $\Delta t$ (positive $\Delta t$ corresponds to $\Delta[F s]_{\mathrm{IMF}}$ leading $\Delta C$ ) which equals 2 months in this case; the line in Fig. $6 \mathrm{~b}$ shows the best-fit regression used to scale the best fit, $\Delta C_{f i t}$, from $\Delta C$. Figure $6 \mathrm{c}$ shows the correlogram giving the cross-correlation function (ccf) between $\Delta[F s]_{\mathrm{IMF}}$ and $\Delta C$ (solid line) and the autocorrelation functions (acf) of $\Delta[F s]_{\mathrm{IMF}}$ (dashed line) and $\Delta C$ (dot-dash line), all shown as a function of lag. Figure $6 \mathrm{~d}$ shows the significance $S_{L}$ of the difference between the ccf and its peak value at the best-fit lag, $\Delta t$, computed as a function of lag using the Fischer-Z test. The uncertainty, $\delta$, is set by the points where $S_{L}$ rises above $90 \%$ and the ccf becomes significantly lower than its peak value. Further details of this correlation procedure are given by Lockwood (2002a). In this case the correlation coefficient $c=0.93$ which, allowing for persistence, is significant at the $S>99.6 \%$ level.

\subsection{Window by window cross-correlation analysis of $C$ and $H$ with $[F s] a a$}

Figures 7 and 8 show the results of applying this procedure to $[F s] a a$ and GCR counts of rigidity exceeding, respectively, $13 \mathrm{GV}$ ( $H$, from the Huncayo/Hawai neutron monitor) and $3 \mathrm{GV}$ ( $C$, from the Climax neutron monitor). The top panel of both figures shows the raw data series, whereas the second panel shows the same data after application of the bandpass filter. Considerable variability can be seen in the GCR counts in the $1-5 \mathrm{yr}$ pass band in the $\Delta C$ and $\Delta H$ series. However, the inherent one year smoothing of $[F s] a a$ is revealed by the relative lack of variation in $\Delta[F s] a a$ (red line in Figs. $7 \mathrm{~b}$ and $8 \mathrm{~b}$ ) when the main solar cycle variation is 

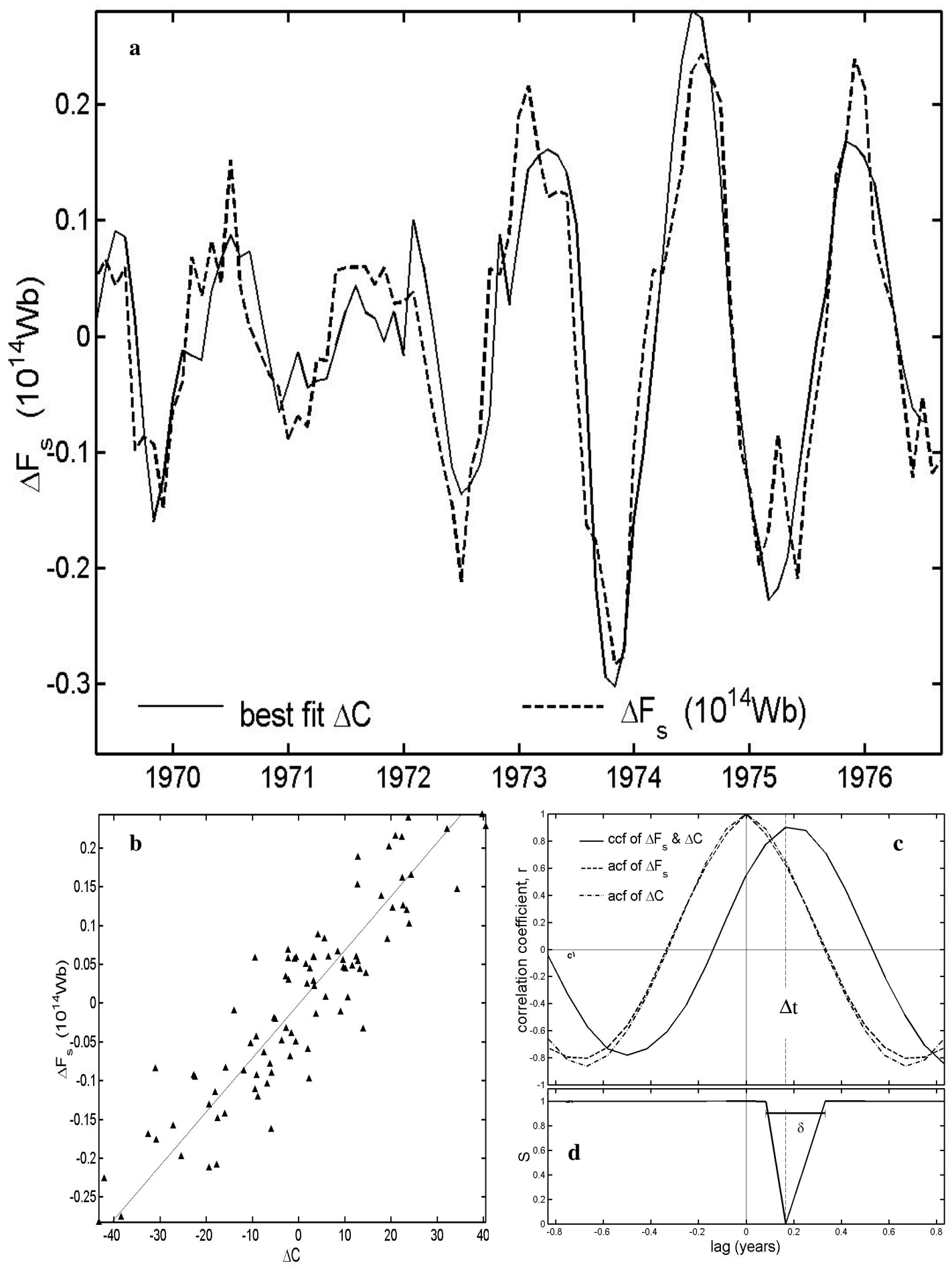

Fig. 6. An example of the correlation analysis between data sets employed in this paper: in this example the filtered Climax cosmic ray counts, $\Delta C$, for 1970-1976 are correlated with the identically filtered open solar flux deduced from near-Earth IMF observations, $\Delta[F s]_{\mathrm{IMF}}$. Tenyear segments of $C$ and $[F s]_{\mathrm{IMF}}$ data are passed through a band-pass filter $(5<T<1 \mathrm{yr})$, and the middle 6 years then analysed. (a) The variations of $\Delta[F s]_{\mathrm{IMF}}$ (dashed line) and the best linear regression fit of $\Delta C_{\mathrm{FIT}}$ (solid line) (b), the scatter plot of $\Delta[F s]_{\mathrm{IMF}}$ against $\Delta C$ for the best-fit lag $\Delta t=2$ months (positive $\Delta t$ corresponds to $\Delta[F s]_{\text {IMF }}$ leading $\Delta C$ ). (c) The correlogram showing the cross-correlation function between $\Delta[F s]_{\mathrm{IMF}}$ and $\Delta C$ (solid line) and the autocorrelation functions of $\Delta[F s]_{\mathrm{IMF}}$ (dashed line) and $\Delta C$ (dot-dash line). (d) The significance $S L$ of the difference between the ccf and its peak value at the best-fit lag, $\Delta t$, computed as a function of lag using the Fischer-Z test. 


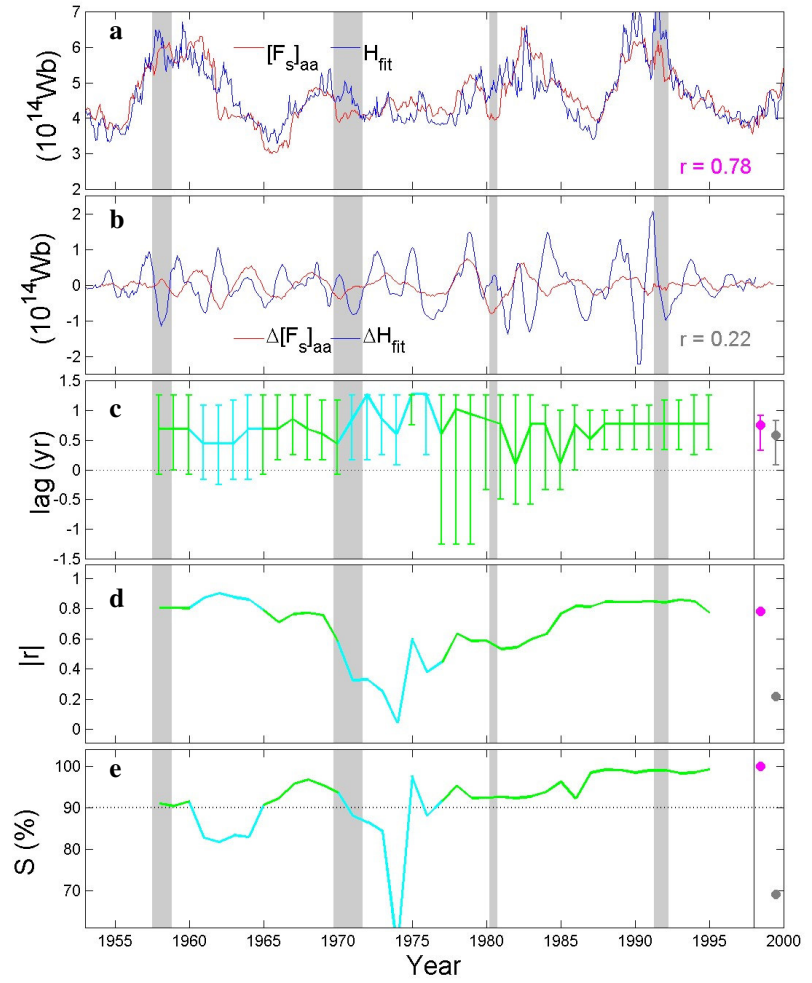

Fig. 7. Results of the correlation analysis of Huancayo/Hawaii neutron monitor ( $>13 \mathrm{GV}$ ) counts $H$ and the open solar flux deduced from the geomagnetic $a a$ index $[F s] a a$. The grey bands mark the times of the reversal in the solar polar field by showing the times between the polar field reversals in the two solar hemispheres. From top to bottom, the panels show: (a) the variations of $[F s] a a$ (red) and $H_{\text {FIT }}$, the best linear regression fit of $H$ to $[F s] a a$ (blue); (b) the band-pass filtered variations $\Delta[F s] a a$ (red) and $\Delta H_{\mathrm{FIT}}$, the best linear regression fit of $\Delta H$ to $\Delta[F s] a a$ (blue); (c) the lag of the peak correlation, $\delta t$; (d) the magnitude of the peak correlation coefficient $|r|$, and (e) the significance of that correlation, $S$. The mauve and the grey data points to the right of (c), (d) and (e) give the values for the full, raw data sequences shown in the top panel (in mauve) and the filtered data sequences (in grey). The correlation of $\Delta[F s] a a$ and $\Delta H$ is low $(r=0.22)$ because of the smoothing inherent $[F s] a a$, so only data points for $[F s] a a$ and $H$ are shown in (c), (d) and (e), computed for each 6-year interval as shown in Fig. 5: data points in green have $S \geq 90 \%$ (the dotted line shown in the bottom panel), those in cyan have $S<90 \%$.

removed after application of the band-pass filter: the shortest period of oscillation is at least 3 years as explained above. In fact, the overall cross-correlation coefficient is very low: $|r|=0.22$ for $\Delta[F s] a a$ with $\Delta H_{\text {FIT }}$ and $|r|=0.18 \Delta[F s] a a$ with $\Delta C_{\mathrm{FIT}}$. We present in Figs. $7 \mathrm{~d}$ and $8 \mathrm{~d}$ the time variations of the cross-correlation coefficients $|r|$ for $[\mathrm{Fs}] a a$ with $\mathrm{H}$ and $\mathrm{C}$ (all unfiltered), respectively. These cross-correlation coefficients have high values $(|r|>0.6)$ with a high significance $(S>80 \%)$ throughout most of the interval where cosmic ray measurements are available. A continuous and significant decrease is seen, however, in both $|r|$ sets between 1968 and 1977, confirming the disagreements already visible

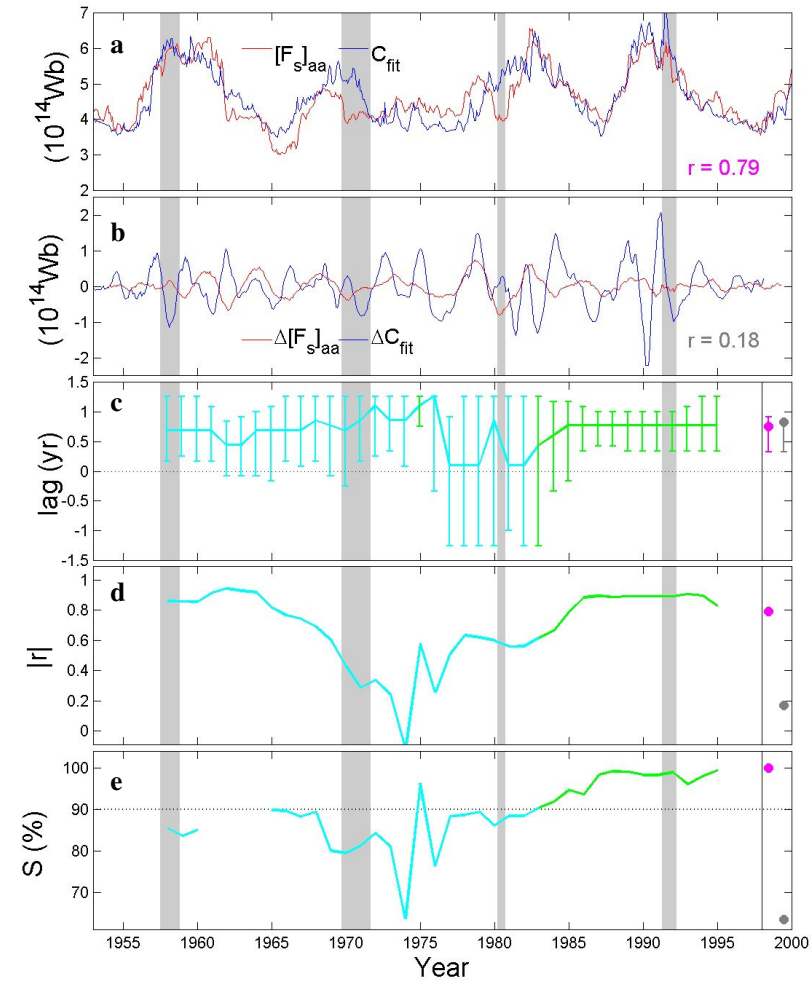

Fig. 8. The same as Fig. 7, for the correlation analysis of Climax neutron monitor $(>3 \mathrm{GV}$ ) counts $C$ and the open solar flux deduced from the geomagnetic $a a$ index $[F s] a a$.

in the raw time series (Fig. 2). The same window-by-window cross-correlation analysis of unfiltered data was carried out with $[F s]_{\mathrm{IMF}}$ instead of $[F s] a a$ and gives very similar results (blue and green lines of Figs. 9 and 10). The differences seen in solar cycle 20 between the variation of the total amount of open field lines in the heliosphere and the GCR count rates at Earth are statistically the same for the two estimates of the total open flux. The middle panels (c) of Figs. 7, 8, 9 and 10 give the lags of peak correlation with uncertainties as derived in Fig. 6. On the right hand-side of panels (c), (d) and (e) of the figures is given respectively, the best fit $\Delta t$, the correlation coefficient $|r|$, and the significance $S$ for the full data sequences: the mauve and grey points are for the unfiltered and the filtered data shown in panels (a) and (b), respectively.

4.3 Window by window cross-correlation analysis of $C$ and $H$ with $[F s]_{\text {IMF }}$, as well as the filtered time series $\Delta C$ and $\Delta H$ with $\Delta[F s]_{\mathrm{IMF}}$

To investigate the imprint of the different oscillations in the open solar flux to the GCR counts further, the window-bywindow cross-correlation procedure was applied to the detrended open solar flux $\Delta[F s]_{\mathrm{IMF}}$ with the detrended cosmic ray counts $\Delta H$ and $\Delta C$. (As mentioned above the inherent smoothing in $[F s]_{a a}$ does not allow us to use it for this analysis). 11-year intervals of data were passed through the same band-pass filter $(5<T<1 \mathrm{yr})$ and, to avoid edge effects, 


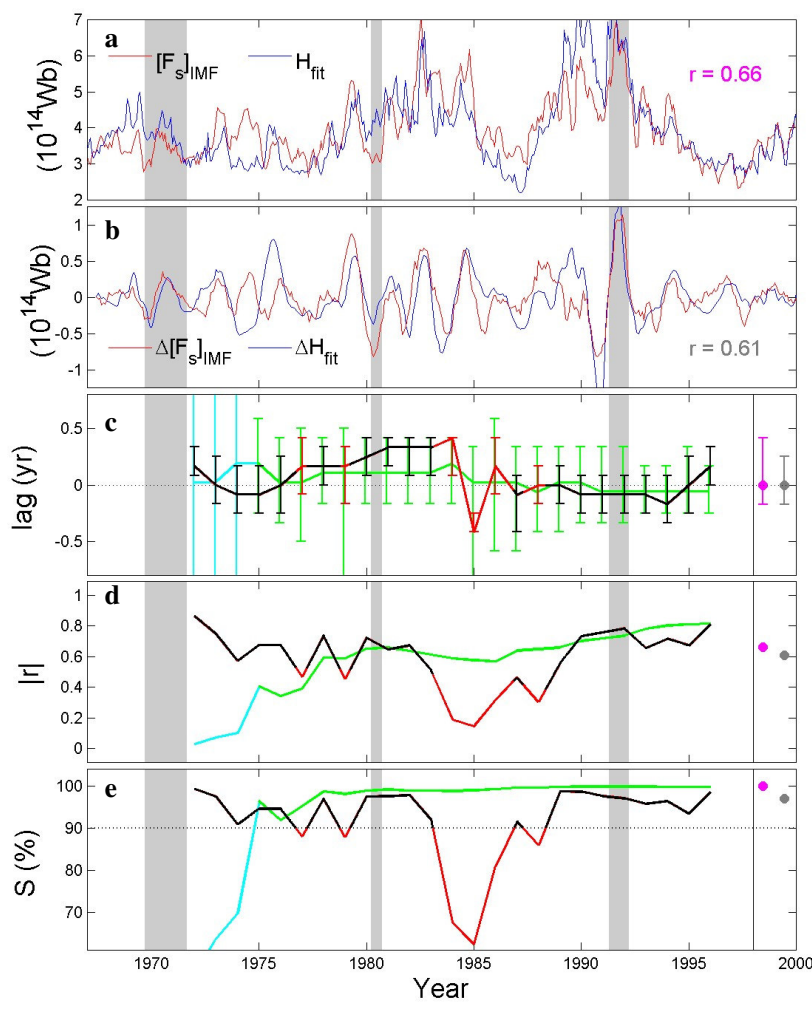

Fig. 9. The same as Fig. 7, for the correlation analysis of Huancayo/Hawaii neutron monitor $(>13 \mathrm{GV})$ counts $H$ and the open solar flux deduced from the radial component of the IMF $[F s]_{\mathrm{IMF}}$. The correlation of $\Delta[F s]_{\mathrm{IMF}}$ and $\Delta H$ is high $(r=0.61)$ so analysis of $\Delta[F s]_{\mathrm{IMF}}$ and $\Delta H$ are shown in (c), (d) and (e) (in addition to that for the unfiltered data, $[F s]_{\mathrm{IMF}}$ and $H$, again shown in green and cyan): those in black have $S \geq 90 \%$ (the dotted line shown in the bottom panel), those in red have $S<90 \%$.

the middle 6 years were then cross-correlated. The results of these cross-correlations are shown in Figs. 9 and 10. The green/light blue lines in parts (c), (d) and (e) are for unfiltered data $C$ and $[F s]_{\mathrm{IMF}}$, the black/red lines are for the filtered data $\Delta[F s]_{\mathrm{IMF}}$ (red) and $\Delta C_{\mathrm{FIT}}$ : the green and black segments are for correlations of significance $S$ exceeding the 90\% threshold (the horizontal dotted line in part e), the red and light blue segments are for $S \leq 90 \%$.

The correlation for the filtered data is strong and significant ( $S>90 \%$, shown in black) at all solar maxima (when the power at 1.68 yrs is greatest, see Fig. 8), as well as the minima for solar field polarity $A>0$ (around 1975 and 1990), but falls to $S<90 \%$ (shown in red) for the minimum with $A<0$ (around 1987). Figure 5 shows that this lack of correlation is not caused by a lack of power in the $1.68 \mathrm{yr}$ oscillation, it being comparable to during the other two minima. It appears that for periods dominated by transient events at solar maximum and for periods dominated by the GCR drifts expected at solar minimum for $q A>0$ (inflows from the heliospheric poles to Earth), the 1.68-yr oscillation in the open solar flux leaves a clear and dominant imprint on the GCR fluxes. However, for the minimum with $q A<0$, when drifts associated

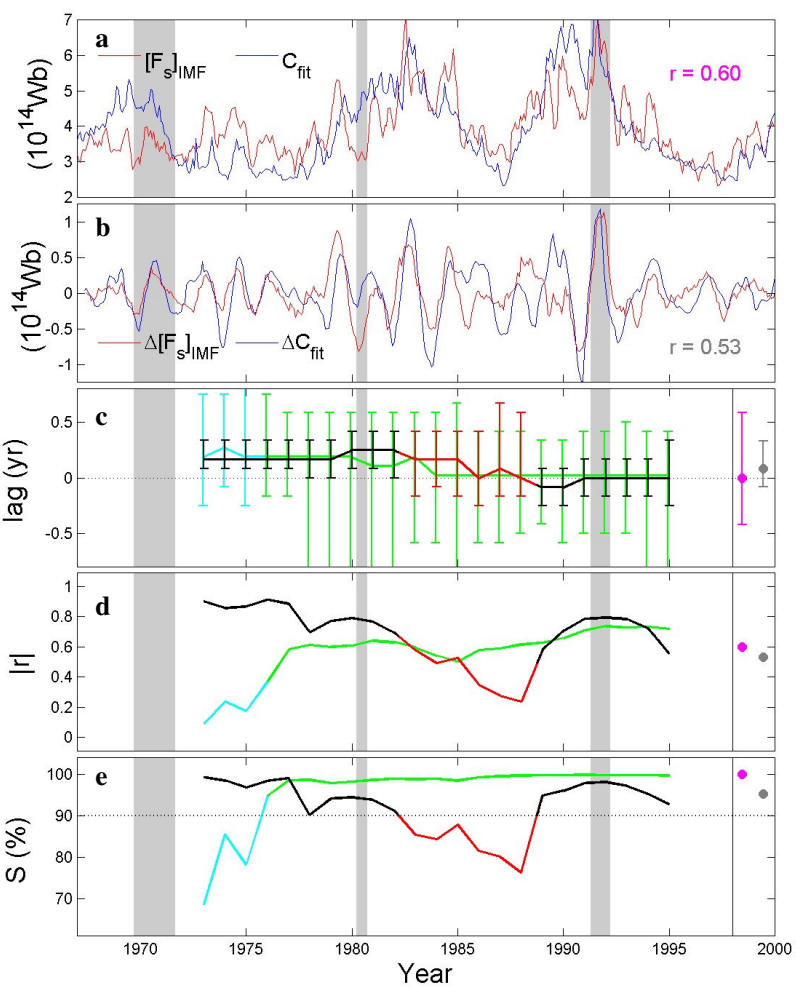

Fig. 10. The same as Fig. 9, for the correlation analysis of Climax neutron monitor ( $>3 \mathrm{GV}$ ) counts $C$ and the open solar flux deduced from the radial component of the IMF $[F s]_{\mathrm{IMF}}$.

with the smooth background field are predicted to force cosmic rays to propagate along the equatorial heliospheric current sheet to Earth the imprint of the 1.68-year oscillation in open flux is considerably weaker and/or more confused. Thus, in the imprint of the 1.68-year variation we seem to find evidence for $q A$-dependent drifts. However, the oscillations detected spectrally in the tilt of the heliospheric current sheet (1982-1995) do not appear in the cosmic ray counts at Earth in this particular period of predicted HCS propagation. An interesting issue is whether these frequencies should or should not appear in the cosmic ray counts according to our present understanding of the propagation of cosmic rays in the heliosphere. The tilt angle has been used in many models of cosmic ray modulation as a time-dependent parameter in order to solve Parker's equation (e.g. Ferreira et al., 2003) or to provide a convenient way of relating the change in the diffusion coefficient to the evolving solar activity (Wibberenz et al., 2002). A spectral analysis of the cosmic ray counts' time series predicted by these various models in the period 1980-1985 would perhaps clarify this point.

The correlation for the filtered data is strong before 1976. This could support suggestions that the earliest IMF data were subject to a drift in their absolute calibration (Belov, 2000) and that this lowers the correlation for unfiltered data (the light blue segments). However, the same low crosscorrelation coefficients are obtained for the open solar flux 
from $a a$ (Figs. 7 and 8), and so it seems that the effect is real and the mechanism linking the total amount of open field lines in the heliosphere to the time-variation of cosmic rays measured at Earth for some reason acted differently in solar cycle 20 (than in solar cycles 19, 21, 22 and 23). Such a hypothesis is valuable considering that solar cycle 20 is characterised by an overall lower activity and a longer magnetic field reversal than in other cycles (Fig. 6). Wibberenz et al. (2002) argue that this apparantly "anomalous" response of the cosmic rays in solar cycle 20 is due to the different times for particles to enter into the depleted regions of the heliosphere by diffusion and drift mechanisms in the two different magnetic polarities of the Sun. Periods of negative polarity $A<0$ when inflows are thought to occur along the HCS (such as the descending phase of solar cycle 20) should be characterised by long recovery processes. The presence of numerous weak field increases, with time lengths less than characteristic recovery times between them, would then be more effective in preventing sunward cosmic rays propagation. This interpretation should, in principle, lead to the GCR depression seen even with the considerably weaker IMF strength of this solar cycle (Fig. 1).

\section{The presence of varying lags between cosmic ray flux at Earth and $\Delta[F s]_{\text {IMF }}$}

The study of lag times between changes in the quantity of open field lines near the Sun (progressively dragged out in the heliosphere by the solar wind) and the subsequent response of cosmic rays is crucial to any approach of GCR solar modulation where diffusion mechanisms have a dominant role.

Figures 7, 8, 9 and 10 show interesting features concerning the time-lag variation between the various cross-correlations of the open solar flux estimates and cosmic ray counts at Earth. In studying these plots it should be remembered that $[F s]_{\mathrm{IMF}}$ leads $[F s] a a$ by $0.75 \pm 0.4$ yr. A lag $(\Delta t>0)$ is expected between open magnetic field and cosmic ray variations because changes in the heliospheric field are carried into the heliosphere at the solar wind speed or less. The mode value of the distribution of the heliographic equator is $370 \mathrm{~km} \mathrm{~s}^{-1}$, which corresponds to distances of the order of 78 AU per year (Hapgood et al., 1991). This agrees well with the typical speeds seen in the streamer belt by Ulysses: the speeds seen by Ulysses in the polar regions are roughly twice this at solar minimum, but at solar maximum the speeds become similar at all latitudes (Richardson et al., 1994). Study of the time-lag for peak correlation between the open solar flux and cosmic ray counts at Earth can be used to infer roughly the locations of main modulation mechanisms associated with the diffusion tensor in the adopted paradigm where changes in the open solar flux are propagated outward at the solar wind speed. A simple look at the size of the error bars in Figs. 7, 8, 9 and 10 shows that the following analysis does not claim to be an exact study of lags; moreover, as pointed out by Dorman et al. (1997, 2001), an accurate interpretation of lags in terms of the size of the modulation region should include the integral modulation effects of all mechanisms known to date. Figures 7c and 8c show the previously discussed results of cross-correlating [ $F s] a a$ with $H$ and $C$, as well as the detrended variations $\Delta[F s] a a$ with $\Delta H$ and $\Delta C$. The best-lag obtained for the overall crosscorrelation is shown as pink and grey dots, respectively, on the right-hand side of the sub-figure. A green/blue line represents the time variation of the best-fit lag obtained for the window by window cross-correlation of $[F s] a a$ with $H$ and $C$. The lag remains in all cases very high at about 8 months with considerable random fluctuations in the period of lowcorrelation coefficients. The time-lag has not changed significantly in the last 40 years and is similar to the overall lag. Noting that $[F s] a a$ leads $[F s]_{\mathrm{IMF}}$ by $0.75 \mathrm{yr}$, Figs. 7 and 8 show no significant evidence for a non-zero lag.

Figures 9c and 10c show the results of cross-correlating $[F s]_{I M F}$ with $H$ and $C$ (greeen/blue lines), as well as the detrended variations $\Delta[F s]_{\mathrm{IMF}}$ with $\Delta H$ and $\Delta C$ (red and black lines). The overall lag obtained for $[F s]_{\mathrm{IMF}}$ $(\Delta t \sim 0)$ and $\Delta[F s]_{\mathrm{IMF}}$ is much smaller than for $[F s] a a$. The green/blue lines in Figs. 9c and 10c show that the timelags between $[F s]_{\mathrm{IMF}}$ and $H$ or $C$ do not remain constant throughout the solar cycles but shift from values close to $0.2 \mathrm{yr}$ in (1971-1981) to very low values (1991-1996) in the $q A>0$ polarity states. The same general trend is noticed in the red/black lines corresponding to the cross-correlation of the detrended variations $\Delta[F s]_{\mathrm{IMF}}$ with $\Delta H$ and $\Delta C$, with additional big fluctuations appearing in the $q A<0$ polarity state (1981-1991) for the cross-correlation with the Hawai/Huncayo neutron monitors counts. These big variations in $q A<0$ are features of the very poor cross-correlation observed during this time period and therefore are artificial (leading to an unphysical point of value below zero, error bars considered). It should be noted that if statistical errors are considered, all other best lag estimates have physical values and the green/blue line values agree with the black/red line values. Around 1980, a persistent value of $\Delta t=0.25 \pm 0.15 \mathrm{yr}$ is observed, in this solar maximum period a solar wind speed of $370 \mathrm{~km} . \mathrm{s}^{-1}$ would lead to a response of cosmic rays to diffusion mechanisms at around 11-30 AU: this is consistent with where merged interaction regions have been seen to act as barriers to GCRs at this time (Burlaga, 1987; Heber et al., 2000). However, at the next solar maximum around 1991 , both the $\Delta[F s]_{\mathrm{IMF}}-\Delta C$ and $\Delta[F s]_{\mathrm{IMF}}-\Delta H$ correlations show a shorter lag, which may be an indicator that the response to changes in the open flux by diffusion is somewhat closer in towards the Sun.

It should be noted that the strong IMF-GCR correlations presented by Cane et al. (1999) and Belov (2000) were for zero lag.

The upper limit to $\Delta t$ for the filtered data places an estimate of the outer limit on the location of high energy GCR scattering processes. Figures $9 \mathrm{c}$ and $10 \mathrm{c}$ show that this upper limit decreased from about $0.3 \mathrm{yr}$ around 1980 to less than $0.1 \mathrm{yr}$ around 1995, the subsequent sunspot minimum with 
$A>0$. This suggests that scattering processes of the inflow, thought to occur mainly over the poles for $A>0$, might have moved inward from within $50 \mathrm{AU}$ to within $10 \mathrm{AU}$. The reasons for this change are not understood. Both $[F s] a a$ and $[F s]_{\text {IMF }}$ (Fig. 1) are of the order of $15 \%$ lower for the second of these sunspot minima. This may allow the GCRs to reach closer to the centre of the heliosphere before they are significantly scattered. The study of these lags will be the subject of following papers. For more information on lags we refer the readers to the extensive work of Dorman et al. (1997, 1999, 2001), who have studied lags between features on the Sun and GCRs by assuming the full integral nature of GCR modulation.

\section{Summary and conclusions}

For all but the period 1968-1975, there is a strong and significant anti-correlation between the open solar flux, as derived from IMF observations or the $a a$ geomagnetic index, and cosmic ray flux at Earth. This is true during most of the solar cycle phases of both magnetic field polarities and could point to a more significant role of the open solar flux in cosmic ray flux modulation than has been attributed to date. We notice an interesting fact, that is, the alternate peaked and rounded maxima seen in the neutron GCR flux time series (giving a 22-year cycle), successfully attributed to the polarity-dependent drift component of the propagation mechanisms of cosmic rays in the heliosphere (see Heber, 2000; Ferreira et al., 2003), is also seen in the variation of the two 1-AU open flux estimates presented here. This implies that the Sun has, as well as a twenty-two-year cycle in the direction of its dipolar field, a twenty-two-year pattern in the quantity of open field lines threading a sphere passing at $1 \mathrm{AU}$. In the last four solar cycles at least, the Sun appears to have reached low open flux values sooner after polarity reversal in $q A>0$ polarity, leading to rounder minima than in $q A<0$ polarity.

In this paper, we have shown that the recently reported 1.68-year variation in GCR fluxes is a persistent feature that is linked to a similar variation in the open solar magnetic flux estimated from the IMF strength at Earth or from the PFSS method using solar magnetograms. This imprint is strongest at solar maximum and cross-correlation analysis shows that there is a difference between $q A>0$ and $q A<0$ cycles at sunspot minimum. Thus, we find possible evidence for the effects of polarity-dependent GCR drifts acting at least 3-4 years around sunspot minimum. The close mapping between the average IMF strength on such different time scales is significant: we confirm the possibility that the variation of the total flux in the heliosphere is a good proxy to the overall variation of diffusion mechanisms affecting the propagation of 3-10 GV cosmic rays, at least in the inner heliosphere. The 1.68-yr variation has only a very weak power for $1982-$ 1986 and is non-existent for the period 1989-1993, in the spectral analysis of the HCS tilt angle. In contrast, the period is present in the GCR data during these time intervals.
A spectral analysis of the cosmic ray counts' time series predicted by the various models which make use of the HCS tilt angle (Ferreira et al., 2003) in the period 1980-1986 would perhaps clarify this point.

Following the submission of this paper, a paper by Kato et al. (2003) has been published in the Journal of Geophysical Research $(108$, A10, 1367) showing the presence of the 1.68year variation in the Voyager cosmic ray data on its way to the outer Heliosphere, with peaks in the power spectral density around the period of 1.68 years also occurring in 1983 and 1992 (Fig. 8 of their paper), thereby demonstrating the global heliospheric nature of these particle flux variations at this time. Their contour map of wavelet power for IMF variations at $1 \mathrm{AU}$ is slightly different from ours, with a single broader enhancement peaking in 1991 (Fig. 9 of their paper). We are currently investigating the origin of these differences (occurring here for different spectral methods).

Acknowledgements. The work of AR is supported by the UK Natural Environment Research Council with the award of a PhD studentship, that of ML by the UK Particle Physics and Astronomy Research Council. The authors are grateful to World Data Centre (WDC) $\mathrm{C} 1$ at RAL, and all scientists who supplied the cosmic ray, geomagnetic, solar and interplanetary data used to the international WDC network. We thank the referee for their comments, which have greatly improved the quality of this paper. We also thank Y.-M. Wang of Naval Research Laboratory, Washington for the provision of the open flux derived from the PFSS method and B. Lanchester for support and encouragement.

Topical Editor R. Forsyth thanks B. Heber and T. Horbury for their help in evaluating this paper.

\section{References}

Balogh, A., Smith, E. J., Tsurutani, B. T., Southwood, D. J., Forsyth, R. J., and Horbury, T. S.: The heliospheric field over the south polar region of the Sun, Science, 268, 1007-1010, 1995.

Belov, A.: Large-scale modulation: view from the Earth, Space Sci. Rev., 93, 79-105, 2000.

Bond, G., Kromer, B., Beer, J., Muscheler, R., Evans, M. N., Showers, W., Hoffman, S., Lotti-Bond, R., Hajdas, I., and Bonani, G.: Persistent solar influence on North Atlantic climate during the Holocene, Science, 294, 2130-2136, 2001.

Burlaga, L. F.: Large-scale fluctuations in B between 13 and 22AU and their effects on cosmic rays, J. Geophys. Res., 92, 13647 $13652,1987$.

Cane, H. V., Wibberenz, G., Richardson, I. G., and von Rosenvinge, T. T.: Cosmic ray modulation and the solar magnetic field, Geophys. Res. Lett., 26, 565-568, 1999.

Clem, J. M. and Evenson, P.: Positron abundance in galactic cosmic rays, Astrophys. J., 568, 216-219, 2002.

Cliver, E. W., Boriakoff, V., and Boumar, K. H.: The 22-year cycle of geomagnetic activity, J. Geophys. Res., 101, 27 091-27 109. 1996.

Couzens, D. A. and King, J. H.: Interplanetary Medium Data Book - Supplement 3, National Space Science Data Center, Goddard Space Flight Center, Greenbelt, Maryland, USA, 1986.

Dorman, L. I., Villoresi, G., Dorman, I. V., Iucci, N., and Parisi, M.: High rigidity CR-SA hysterisis phenomenon and dimension 
of modulation region in the heliosphere in dependence of particle rigidity, Proc. 25th Intern. Cosmic Ray Conf., 2, 69-72, 1997.

Dorman, L. I., Dorman, N., Iucci, N., Parisi, M., and Villoresi, G.: Hysterisis phenomenon: the dimension of modulation region in dependence of cosmic ray energy, Proc. 26th Intern. Cosmic Ray Conf., 7, 194-197, 1999.

Dorman, L. I., Iucci, N., and Villoresi, G.: Time lag between cosmic ray and solar activity: solar minimum of 1994-1996 and residual modulation, Adv. Space Res., 27, 3, 595-600, 2001.

Droge, W.: Solar particle transport in a dynamical quasi-linear theory, Astrophys. J., 589, 1027-1039, 2003.

Dyer, C. S. and Truscott, P. R.: Cosmic radiation effects on Avionics, Radiat. Prot. Dosim., 337-342, 1999.

Evenson, P.: Cosmic ray electrons, Space Sci. Rev., 83, 63-73, 1988.

Ferreira, S. E. S., Potgieter, M. S., Heber, B., and Fichtner, H.: Charge-sign dependent modulation in the heliopshere over a 22year cycle., Ann. Geophys., 21, 1359-1366, 2003.

Fisk, L. A.: An overview of the transport of galactic and anomalous cosmic rays in the heliopshere: theory, Ad. Space Res., 23, 3, 415-423, 1999.

Gazis, P. R.: Solar cycle variation of the heliosphere, Rev. Geophys., 34, 379-402, 1996.

Ginzburg, V. L.: Cosmic Ray astrophysics (history and general review), Physics-Uspekhi, 39 (2), 155-168, 1996.

Gnevyshev, M. N.: On the 11-years cycle of solar activity, Sol. Phys., 1, 107-120, 1967.

Gnevyshev, M. N.: Essential features of the 11-year solar cycle, Sol. Phys., 51, 175-183, 1977.

Goldstein, B. E.: Ulysses observations of solar wind plasma parameters in the ecliptic from 1.4 to $4.5 \mathrm{AU}$ and out of the ecliptic, Space Sci. Rev., 72, 113-116, 1994.

Hapgood, M. A., Bowe, G., Lockwood, M., Willis, D. M., and Tulunay, Y.: Variability of the interplanetary magnetic field at 1 A.U. over 24 years: 1963-1986, Planet. Space Sci., 39, 411-423, 1991.

Harrison, R. G.: Long-term measurements of the global atmospheric electric circuit at Eskdalemuir, Scotland, 1911-1981, Atmos. Res., 70(1), 1-19, 2003.

Heber, B., Ferrando, P., Raviart, A., Wibberenz, G., Müller-Mellin, R., Kunow, H., Sierks, H., Bothmer, V., Posner, A., Paizis, C., and Potgieter, M. S.: Differences in the temporal variations of galactic cosmic ray electrons and protons: implications from Ulysses at solar minimum, Geophys. Res. Lett., 26, 2133-21236, 1999.

Heber, B., Clem, J. M., Müller-Mellin, R., Kunow, H., Ferreira, S. E. S., and Potgieter, M. S.: Evolution of the galactic cosmic ray electron to proton ratio: Ulysses COSPIN?KET observations, Geophys. Res. Lett., 30, 19, 8032-8045, 2003.

Hedgecock, P. C.: Measurements of the interplanetary magnetic field in relation to the modulation of cosmic rays, Solar Phys., 42, 497-527, 1975.

Howe, R., Christensen-Dalsgaard, J., Hill, F., Komm, R. W., Larsen, R. M., Schou, J., Thompson, M. J., and Toomre, J.: Dynamic Variations at the base of the convection zone, Science, 287, 2456-2460, 2000.

Jokipii, J. R., Levy, E. H., and Hubbard, W. B.: Effects of particle drift on cosmic ray transport. I. General properties, application to solar modulation, Astrophys. J., 213, 861-868, 1977.

Kay, S. M. and Marple, S. L.: Spectrum analysis: a modern perspective, Proc. IEEE, 69, 1380-1419, 1981.

Kudela, K., Ryback, J., Antalova, A., and Storini, M.: Time evolu- tion of low frequency periodicities in cosmic ray intensity, Sol. Phys. 205, 165-175, 2002.

Lockwood, M.: Long-Term Variations in the Magnetic Fields of the Sun and the Heliosphere: their origin, effects and implications, J. Geophys. Res., 106, 16 021-16038, 2001.

Lockwood, M.: An evaluation of the correlation between open solar flux and total solar irradiance, Astron. and Astrophys., 382, 6786361, 2002a.

Lockwood, M.: Long-term variations in the open solar flux and links to variations in earth's climate, in: From Solar Min to Max: Half a solar cycle with SoHO, Proc. SoHO 11 Symposium, Davos, Switzerland, March 2002, ESA-SP-508, pp 507522, ESA Publications, Noordvijk, The Netherlands, 2002b.

Lockwood, M.: Twenty-three cycles of changing open solar flux, J. Geophys. Res., 108, A3, 1128-1143, 2003.

Lockwood, M. and Stamper, R.: Long-term drift of the coronal source magnetic flux and the total solar irradiance, Geophys. Res. Lett., 26, 2461-2464, 1999.

Lockwood, M., Stamper, R., and Wild, M. N.: A doubling of the sun's coronal magnetic field during the last 100 years, Nature, 399, 437-439, 1999a.

Lockwood, M., Stamper, R., Wild, M. N., Balogh, A., and Jones, G.: Our changing sun, Astron. and Geophys., 40, 4.10-4.16, 1999b.

Lockwood, M., Forsyth, R. B., Balogh, A., and McComas, D. J.: Open solar flux estimates from the near-Earth measurements of the Interplanetary magnetic field: comparison of the first two perihelion passes of the Ulysses spacecraft, Ann. Geophys., 13951405, 2004.

Maravilla, D., Lara, A., Valdés-Galicia, J. F., and Mendoza, B.: An Analysis of Polar Coronal Hole Evolution: Relations to Other Solar Phenomena and Heliospheric Consequences, Solar Physics, 203 (1), 27-38, 2001.

Marsh, N. and Svensmark, H.: Cosmic rays, clouds and climate, Space Science Rev., 94, 215-230, 2000.

Mayaud, P. N.: The $a a$-indices: A 100-year series characterising the magnetic activity, J. Geophys. Res., 77, 6870-6874, 1972.

McDonald, F. B, Trainer, J. H., Trainer, J. D., Mihalov, J. H., Wolfe, J. H., and Webber, W. R.: Radially propagating shock waves in the outer heliosphere: The evidence from Pioneer 10 energetic particle and plasma observations, Astrophys. J., 246, L165, 1981.

McDonald, F. B., Lal, N., and McGuire, R. E.: The role of drifts and global merged interaction regions in the long-term modulation of cosmic rays, J. Geophys. Res., 98, 1243-1256, 1993.

McIntosh, P. S., Thompson, R. J., and Willcok, E. C.: A 600-day periodicity in coronal holes, Nature, 360, 322, 1992.

Moraal, H., Steenberg, C. D., and Zank, G. P.: Simulations of galactic and anomalous cosmic ray transport in the heliosphere, Adv. Space Res., 23, 3, 425-436, 1999.

Mursula, K. and Zieger, B.: Simultaneous occurrence of mid-term periodicities in solar wind speed, geomagnetic activity and cosmic rays, 26th ICRC Proceedings, SH 3.2.11, 1999.

Mursula, K. and Zieger, B.: The 1.3-year variation in solar wind speed and geomagnetic activity, Adv. Space Res., 25 (9), 1939 1942, 2000.

Neff, U., Burns, S. J., Mangini, A., Mudelsee, M., Fleitmann, D., and Matter, A.: Strong coherence between solar variability and the Monsoon in Oman between 9 and 6 kyrs ago, Nature, 411, 290-293, 2001.

Nagashima, K., Sakakibara, S., Fenton, A. G., and Humble, J. E.: The insensitivity of the cosmic ray galactic anisotropy to heliosmagnetic polarity reversals, Plan. Space Sci., 33, 395-405, 1985. 
Parhi, S., Burger, R. A., Bieber, J. W., and Matthaeus, W. H.: Challenges for an 'ab-initio' theory of cosmic ray modulation, Proceedings of ICRC, 3670, 2001.

Parker, E. N.: The passage of energetic charged particles through interplanetary space, Planet. Space Sci., 13, 9-49, 1965.

Perko, J. S. and Fisk, L. A.: Solar modulation of galactic cosmic rays. V - Time-dependent modulation, J. Geophys. Res., 88, 9033-9036, 1983.

Potgieter, M. S. and Ferreira, S. E. S.: Modulation of cosmic rays in the heliosphere over 11 and 22 year cycles: A modeling perspective, Adv. Space Res., 27 (3), 481-492, 2001.

Richardson, J. D., Paularena, K. I., Belcher, J. W., and Lazarus, A. J.: Solar wind oscillations with a 1.3 year period, Geophys. Res. Lett., 21, 1559-1560, 1994.

Russell, C. T. and McPherron, R. L.: The semi-annual variation of geomagnetic activity, J. Geophys. Res., 78, 92-115, 1973.

Shea, M. A. and Smart, D. F.: Cosmic ray implications for human health, Space Sci. Rev., 93, 187-205, 2000.

Smith, E. J. and Balogh, A.: Ulysses observations of the radial magnetic field, Geophys. Res. Lett., 22, 3317-3320, 1995.

Silverman, S. M. and Shapiro, R.: Power spectral analysis of auroral occurrence frequency, J. Geophys. Res., 88, 6310-6316, 1983.

Stamper, R., Lockwood, M., Wild, M. N., and Clark, T. D. G.: Solar causes of the long term increase in geomagnetic activity, $\mathrm{J}$. Geophys. Res., 104, 28 325-28 342, 1999.

Storini, M., Pase, S., Sykora, J., and Parisi, M.: Two components of cosmic ray modulation, Solar Phys., 172, 317-325, 1997.
Suess, S. T. and Smith, E. J.: Latitudinal dependence of the radial IMF component-coronal imprint, Geophys, Res. Lett., 23, 3267 3270, 1996.

Valdés-Galicia, J. F. and Mendoza, B.: On the role of large scale solar photospheric motions in the cosmic-ray 1.68-yr intensity variation, Solar Phys., 178, 183-191, 1998.

Valdés-Galicia, J. F., Pérez-Enríquez, R., and Otaola, J. A.: The cosmic ray 1-68-year variation: a clue to understand the nature of the solar cycle?, Solar Phys., 167, 409-417, 1996.

Vasyluinas, V. M., Kan, J. R., Siscoe, G. L., and Akasofu, S. I.: Scaling relations governing magnetospheric energy transfer, Planet. Space. Sci., 30, 359-365, 1982.

Wang, Y.-M. and Sheeley Jr., N. R.: Solar implications of Ulysses interplanetary field measurements, Astrophys. J., 447, L143L146, 1995.

Wang, Y.-M. and Sheeley Jr., N. R.: On the fluctuating component of the sun's large-scale magnetic field, Astrophys. J., 590, 1111$1120,2003$.

Wibberenz, G. and Cane, H. V.: Simple analytical solutions for propagating diffusive barriers and application to the 1974 minicycle, J. Geophys. Res., 105, 18 315-18 325, 2000.

Wibberenz, G., Richardson, I. G., and Cane, H. V.: A simple concept for modelling cosmic ray modulation in the inner heliosphere during solar cycles 20-23, J. Geophys. Res., 107, 13531368, 2002. 